\author{
FAST COMMUNICATION
}

\title{
DERIVATION OF CONTINUUM MODELS FOR THE MOVING CONTACT LINE PROBLEM BASED ON THERMODYNAMIC PRINCIPLES*
}

\author{
WEIQING REN ${ }^{\dagger}$ AND WEINAN $E^{\ddagger}$
}

\begin{abstract}
Contact lines arise as the boundaries of free boundaries in fluids. This problem is interesting and important, not only because it arises in many applications, but also because of the distinct mathematical and physical features it has, such as singularities, hysteresis, instabilities, competing scaling regimes, etc. For a long time, this area of study was plagued with conflicting theories and uncertainties regarding how the problem should be modeled. In the present paper we illustrate how continuum models for the moving contact line problem can be derived using simple thermodynamic considerations. Both the sharp interface models and diffuse interface models are derived.
\end{abstract}

Key words. Moving contact lines, molecular dynamics, thermodynamics.

AMS subject classifications. 76D27, 76D45, 76T99.

\section{Introduction}

The purpose of this paper is to derive continuum models for contact line dynamics, based on thermodynamic principles. A contact line is the intersection of three phases, often two fluid phases and a solid phase. The two fluid phases can either be two immiscible fluids, such as water and oil, or the two phases of the same substance such as the vapor and liquid phases of water. The solid phase is usually the container for the fluids. For this reason, contact lines are also the boundaries of the free boundary between the two fluid phases, and is therefore an ubiquitous part of interfacial phenomena.

The contact line problem is interesting and important, not only for the fact that it arises in many applications such as coating, printing, porous medium flows, and in many micro-fluidic devices, but also because of its theoretical interest; for example, singularities, hysteresis, etc $[1,2,3,4,5,6]$.

It is well-known that classical hydrodynamics (i.e. the Navier-Stokes equation and the no-slip boundary condition) predicts a non-integrable singularity for the viscous stress $[7,8]$. In an effort to remove the singularity, many different continuum models have been proposed to regularize the model. All of these models introduce an inner region near the contact line. However, the proposed physical scenarios inside the inner regions are quite different for the different models. Some typical ideas include:

- The introduction of slip regions, in which the no-slip boundary condition is replaced either by the Navier boundary condition or by a prescribed slip profile, or the stress-free condition $[3,9,10,11]$.

- Diffuse interface models $[12,13,14,15,16,17]$.

*Received: August 17, 2010; accepted (in revised version): September 23, 2010. Communicated by Shi Jin.

${ }^{\dagger}$ Courant Institute of Mathematical Sciences, New York University, New York, NY 10012, USA (weiqing@cims.nyu.edu).

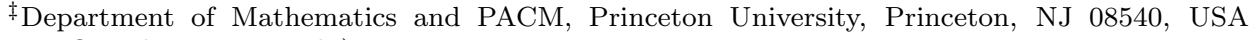
(weinan@math.princeton.edu). 


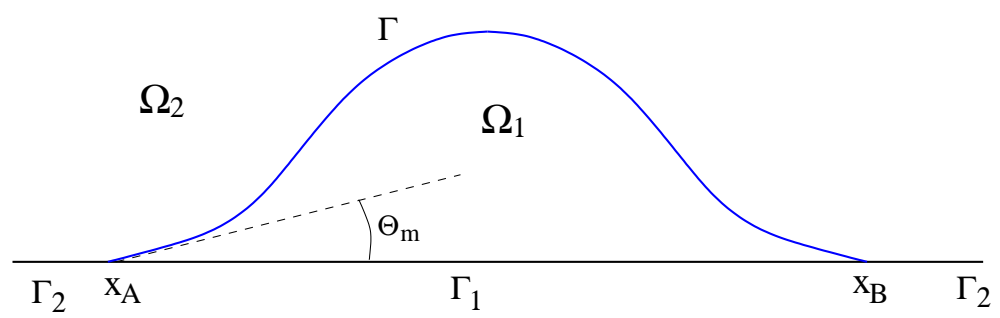

FIG. 2.1. A droplet on a substrate. $x_{A}$ and $x_{B}$ are the contact lines; $\theta_{m}$ is the contact angle at the wall; $\Gamma_{1}, \Gamma_{2}$ and $\Gamma$ denote the fluid-solid and fluid-fluid interfaces, respectively.

Another type of model is based on the molecular kinetic theory in which the contact line motion is modeled by an activated process $[18,19]$. As we see from the work of Cox and Jacqmin [11, 13], it is difficult to differentiate these models from their predictions on the macroscopic flow behavior and apparent contact angle. This also means that it is difficult to use experimental results to validate these models. To find out what the right model should be, we have to look elsewhere.

Two complementary approaches have been taken to find the right continuum models. One approach is to extract the right model from detailed molecular dynamics studies $[20,21,22,23,14,24]$. In this direction, the most detailed and most systematic work was done by Qian et al. and their result is formulated as a diffuse interface model [14]. As an alternative approach, we ask the question, in the spirit of generalized thermodynamics [25]:

What is the simplest form of boundary condition that is consistent with the 2nd law of thermodynamics?

We will work under the assumption that the system is iso-thermal. Therefore the second law of thermodynamics simply states that the free energy can not increase. It is obvious that the philosophy described here works equally well for the more general case when temperature varies in the system. In that case, one has to work with the entropy instead of the free energy.

Beginning with an expression for the free energy of the system, the standard procedure of generalized thermodynamics consists of the following two steps:

- Step 1. Identify all the sources of free energy dissipation. Each contribution is expressed in the form of the product of a generalized force and the corresponding generalized flux. Along the way, one also identifies the reversible fluxes - the component of the fluxes that does not contribute to the dissipation.

- Step 2. Relate the generalized fluxes to the generalized forces.

This simple tool is remarkably effective for the problem at hand, as we see below.

The present paper is limited to the derivation of the models. More detailed discussions on these continuum models and their applications will be presented elsewhere $[26]$.

\section{A sharp-interface contact line model}

Consider the spreading of a liquid droplet on a solid surface (see Figure 2.1). The most important physical parameters are the three surface tension coefficients, $\gamma_{1}, \gamma_{2}$ and $\gamma$, where $\gamma$ is the fluid-fluid surface tension, $\gamma_{1}$ and $\gamma_{2}$ are the surface tension parameters between the solid and the two fluid phases. The spreading parameter $S$ 
is defined as

$$
S=\gamma_{2}-\left(\gamma_{1}+\gamma\right)
$$

$S \geq 0$ corresponds to the case of complete wetting, and $S<0$ corresponds to the case of partial wetting. In the case of partial wetting, the static contact angle $\theta_{Y}$ is determined by the Young's relation:

$$
\gamma_{2}-\gamma_{1}=\gamma \cos \theta_{Y}
$$

To begin with, let us first note that existing evidence suggests that for simple fluids, the accuracy of the linear constitutive relation in the bulk seems quite adequate; even though the viscous stresses near the contact line can be quite large, they are quite far from being large enough to cause significant deviation from the linear response regime. This issue was examined quite carefully in the work of Qian et al [14]. Therefore, we will assume that the standard Navier-Stokes equations hold in the bulk $\Omega_{i}, i=1,2$ :

$$
\begin{aligned}
\rho_{i}\left(\partial_{t} \mathbf{u}+\mathbf{u} \cdot \nabla \mathbf{u}\right) & =-\nabla p+\nabla \cdot \tau_{d}, \\
\nabla \cdot \mathbf{u} & =0,
\end{aligned}
$$

and

$$
\tau_{d}=\eta_{i}\left(\nabla \mathbf{u}+(\nabla \mathbf{u})^{T}\right) .
$$

Here $\eta_{i}$ 's are the viscosities of the fluids. We will also neglect any complications on the fluid-fluid interface, and assume that the standard Laplace-Young interfacial condition holds. Therefore our focus will be on the boundary conditions at the solid surface.

The total free energy of the system can be written as:

$$
E=\sum_{i=1,2} \int_{\Omega_{i}} \frac{1}{2} \rho_{i}|\mathbf{u}|^{2} d \mathbf{x}+\left(\gamma_{1}-\gamma_{2}\right)\left|\Gamma_{1}\right|+\gamma|\Gamma|+\int_{\Gamma_{1}} V(h) d \sigma,
$$

where the first term is the kinetic energy, the second and third terms are the interfacial energy $\left(\left|\Gamma_{1}\right|\right.$ and $|\Gamma|$ are the length of the interfaces), and the last term is the energy due to long-range molecular interactions between the solid substrate and the fluids $[27,2]$, where $h$ is the height of the droplet. A straightforward calculation gives, in two dimensions,

$$
\begin{aligned}
\frac{d E}{d t}= & -\sum_{i=1,2} \int_{\Omega_{i}} \eta_{i}|\nabla \mathbf{u}|^{2} d \mathbf{x}+\sum_{i=1,2} \int_{\Gamma_{i}}\left(\mathbf{t} \cdot \tau_{d} \cdot \mathbf{n}\right) u_{s} d \sigma \\
& +\left(\gamma\left(\cos \theta_{m}-1\right)-S+V(0)\right) u_{\ell}
\end{aligned}
$$

where we have used the dynamic equations in (2.3), the constitutive relation (2.4), and the following interface conditions at the fluid interface:

$$
[p]-V^{\prime}(h)+\gamma \kappa=\mathbf{n} \cdot\left[\tau_{d}\right] \cdot \mathbf{n}, \quad \mathbf{t} \cdot\left[\tau_{d}\right] \cdot \mathbf{n}=0,
$$

where $\kappa$ is the curvature of the fluid interface, $\mathbf{n}$ and $\mathbf{t}$ are the unit normal vector and the unit tangent vector to the fluid interface, respectively. In $(2.6), u_{s}$ is the slip 
velocity of the fluids at the wall (for simplicity we have assumed the wall is at rest), $u_{\ell}$ is the (outward) normal velocity of the contact line, $\theta_{m}$ is the dynamic contact angle, $\mathbf{t}$ and $\mathbf{n}$ are the unit tangent vector and the unit normal to the solid surface. Similar results can be obtained in three dimensions. In [28], the form of the energy dissipation (2.6) was used to derive the condition for the dynamic contact angle in the case when there is no energy dissipation at the contact line.

Let us focus at the fluid-solid interfaces $\Gamma_{1}$ and $\Gamma_{2}$. There, we have the standard no-penetration condition $\mathbf{u} \cdot \mathbf{n}=0$. In the language of the generalized thermodynamics [25], the slip velocity $u_{s}$ and the contact line velocity $u_{\ell}$ are the generalized fluxes, $\tau_{s}=\mathbf{t} \cdot \tau_{d} \cdot \mathbf{n}$ and $\tau_{Y}=\gamma\left(\cos \theta_{m}-1\right)-S+V(0)$ are the generalized forces. The next step in the generalized thermodynamics formalism is to relate the generalized forces to the generalized fluxes. These relations are the constitutive relations. We will assume the simplest form of the constitutive relations, namely that the generalized forces are local functions of the generalized fluxes. This gives us

$$
\begin{aligned}
\mathbf{t} \cdot \tau_{d} \cdot \mathbf{n} & =f_{s}\left(u_{s}\right), \\
\gamma\left(\cos \theta_{m}-1\right)-S+V(0) & =f_{\ell}\left(u_{\ell}\right),
\end{aligned}
$$

where the two functions $f_{s}$ and $f_{\ell}$ have to be determined by other means, for example, molecular dynamics simulation or experimental measurements. The second law of thermodynamics requires that

$$
u \cdot f_{s}(u) \leq 0, \quad u \cdot f_{\ell}(u) \leq 0 .
$$

Equations (2.8a) and (2.8b) are certainly not the most general form of constitutive relations one can have. But they are the simplest, and for now, we will stick with this form.

Equation (2.8a) is the boundary condition for the slip velocity $u_{s}$ at the solid surface. When $f_{s}$ is linear, it reduces to the well-known Navier boundary condition:

$$
\mathbf{t} \cdot \tau_{d} \cdot \mathbf{n}=-\beta u_{s}
$$

where $\beta$ is the friction coefficient.

Equation $(2.8 \mathrm{~b})$ is the boundary condition for the contact angle $\theta_{m}$. In the case of partial wetting $(S<0)$ it is a common practice to neglect the surface potential $V(h)$, so that Equation $(2.8 \mathrm{~b})$ reduces to

$$
\gamma\left(\cos \theta_{m}-\cos \theta_{Y}\right)=f_{\ell}\left(u_{\ell}\right),
$$

where $\theta_{Y}$ is the static contact angle satisfying the Young's relation (2.2). This special case when $f_{\ell}$ is linear has been discussed in [24].

In the case of complete wetting $(S \geq 0)$, the disjoining pressure is essential for the thin film dynamics. In the case when $S=V(0)$ [2], one may note that the left-hand side of $(2.8 \mathrm{~b})$ becomes $\tau_{Y}=\gamma\left(\cos \theta_{m}-1\right)$, which is non-positive. The non-positivity condition in (2.9) requires that

$$
\left(\cos \theta_{m}-1\right) \cdot u_{\ell} \leq 0
$$

from which we conclude that

$$
\text { 1) } u_{\ell} \geq 0 ; \text { or 2) } \theta_{m}=0 \text { if } u_{\ell}<0 \text {. }
$$


The latter case corresponds to the situation when the droplet recedes. Therefore, when the droplet recedes, the dynamic contact angle $\theta_{m}$ has to vanish. To summarize, in the case of complete wetting the boundary condition $(2.8 \mathrm{~b})$ becomes

$$
\gamma\left(\cos \theta_{m}-1\right)=\left\{\begin{array}{cc}
f_{\ell}\left(u_{\ell}\right), & \text { if } u_{\ell} \geq 0 \\
0, & \text { if } u_{\ell}<0
\end{array}\right.
$$

When the friction law is linear and the contact angle is small, the above equation further reduces to

$$
\frac{1}{2} \gamma \theta_{m}^{2}=\left\{\begin{array}{cc}
\beta^{*} u_{\ell}, & \text { if } u_{\ell} \geq 0 \\
0, & \text { if } u_{\ell}<0
\end{array}\right.
$$

where $\beta^{*}$ is the friction coefficient at the contact line, and it has the dimension of viscosity.

The boundary condition (2.14) is derived from (2.8b) under the assumption $V(0)=S$. In the general situation when $V(0)$ and $S$ are two independent parameters [29], both contribute to the driving force for the contact line motion. The above argument still applies but the boundary conditions need to be modified.

To summarize, the sharp-interface model derived above consists of the NavierStokes equations (2.3) and (2.4), the interface conditions in (2.7), and the boundary conditions in (2.8) for $u_{s}$ and $\theta_{m}$. The functions $f_{s}$ and $f_{\ell}$ still need to be specified for the model to be complete. In [26], these functions were computed from molecular dynamics.

\section{Diffuse interface models}

Next we apply the same methodology as used in the last section to derive diffuse interface models for moving contact lines. Diffuse interface models have been used for the computation of flows associated with complex interface morphologies and topological changes (see [30] for a review), and have also been used to model moving contact lines $[12,13,14,15,16,17]$. Here our main purpose is to derive the boundary conditions for the phase-field models. We will distinguish two cases. We first consider the dynamics of one-component fluids (e.g. a liquid-vapor system) on solid surfaces. Then we discuss two-component fluids.

3.1. One-component fluids. Let $\rho$ denote the mass density of the fluid. The total free energy is written as

$$
\begin{aligned}
E(t)= & \int_{\Omega}\left(\frac{1}{2} \kappa|\nabla \rho|^{2}+f(\rho)+\rho V^{\prime}(y)\right) d \mathbf{x} \\
& +\int_{\Omega} \frac{1}{2} \rho|\mathbf{u}|^{2} d \mathbf{x}+\int_{\partial \Omega}\left(\gamma_{w f}(\rho)+\rho V(0)\right) d \sigma \\
= & E_{p}+E_{k}+E_{s},
\end{aligned}
$$

where the three terms represent the potential energy $\left(E_{p}\right)$, the kinetic energy $\left(E_{k}\right)$ and the surface energy $\left(E_{s}\right)$, respectively; $f(\rho)$ is a double well potential with two minima at $\rho=1$ and $\rho=0$ corresponding to the liquid phase and the vapor phase, respectively; $V(y)$ is the surface potential at $y$ - the coordinate normal to the solid wall; $\gamma_{w f}$ is the interfacial energy between the fluid and the solid. 
Define the material derivative $D_{t}=\partial_{t}+\mathbf{u} \cdot \nabla$. The equations in the bulk are written in the form of compressible fluids:

$$
\begin{array}{r}
D_{t} \rho=-\rho \nabla \cdot \mathbf{u}, \\
\rho D_{t} \mathbf{u}=\nabla \cdot \tau+\mathbf{J}_{\mathbf{u}},
\end{array}
$$

where $\tau$ is the viscous stress and is assumed to be linear:

$$
\tau=\nu\left(\nabla \mathbf{u}+\nabla \mathbf{u}^{T}\right)+\lambda(\nabla \cdot \mathbf{u}) .
$$

$\mathbf{J}_{\mathbf{u}}$ consists of non-dissipative forces which will be determined below.

Define the chemical potential:

$$
\mu=-\kappa \Delta \rho+f^{\prime}(\rho) .
$$

Then using the equations in (3.2), we obtain

$$
\begin{aligned}
\frac{d E_{p}}{d t} & =\int_{\Omega}\left(-\kappa \Delta \rho+f^{\prime}(\rho)+V^{\prime}(y)\right) \partial_{t} \rho d \mathbf{x}+\int_{\partial \Omega} \kappa \partial_{\mathbf{n}} \rho \partial_{t} \rho d \sigma \\
& =-\int_{\Omega}\left(\mu+V^{\prime}(y)\right) \nabla \cdot(\rho \mathbf{u}) d \mathbf{x}+\int_{\partial \Omega} \kappa \partial_{\mathbf{n}} \rho \partial_{t} \rho d \sigma \\
& =\int_{\Omega} \rho \mathbf{u} \cdot \nabla\left(\mu+V^{\prime}(y)\right) d \mathbf{x}+\int_{\partial \Omega} \kappa \partial_{\mathbf{n}} \rho \partial_{t} \rho d \sigma, \\
\frac{d E_{k}}{d t} & =\int_{\Omega} \rho \mathbf{u} \cdot D_{t} \mathbf{u} d \mathbf{x} \\
& =\int_{\Omega} \mathbf{u} \cdot\left(\nabla \cdot \tau+\mathbf{J}_{\mathbf{u}}\right) d \mathbf{x} \\
& =\int_{\Omega}\left(-\nabla \mathbf{u}: \tau+\mathbf{u} \cdot \mathbf{J}_{\mathbf{u}}\right) d \mathbf{x}+\int_{\partial \Omega} \mathbf{u} \cdot(\tau \mathbf{n}) d \sigma, \\
\frac{d E_{s}}{d t} & =\int_{\partial \Omega}\left(\gamma_{w f}^{\prime}(\rho)+V(0)\right) \partial_{t} \rho d \sigma .
\end{aligned}
$$

Therefore,

$$
\begin{aligned}
\frac{d E}{d t}= & -\int_{\Omega} \nabla \mathbf{u}: \tau d \mathbf{x}+\int_{\Omega} \mathbf{u} \cdot\left(\mathbf{J}_{\mathbf{u}}+\rho \nabla\left(\mu+V^{\prime}(y)\right)\right) d \mathbf{x} \\
& +\int_{\partial \Omega}\left(\mathcal{B}[\rho] \partial_{t} \rho+\mathbf{u} \cdot \tau \mathbf{n}\right) d \sigma
\end{aligned}
$$

where in the last integral the operator $\mathcal{B}[\rho]$ is defined as

$$
\mathcal{B}[\rho]=\kappa \partial_{\mathbf{n}} \rho+\gamma_{w f}^{\prime}(\rho)+V(0) .
$$

On $\partial \Omega, \rho$ satisfies an equation of the form

$$
\partial_{t} \rho+\left(\mathbf{u}_{s} \cdot \nabla_{s}\right) \rho=J_{b},
$$


where $\mathbf{u}_{s}$ is the slip velocity on $\partial \Omega, J_{b}$ is a dissipative contribution yet to be determined. Then from (3.6) we obtain

$$
\begin{aligned}
\frac{d E}{d t}= & -\int_{\Omega} \nabla \mathbf{u}: \tau d \mathbf{x}+\int_{\Omega} \mathbf{u} \cdot\left(\mathbf{J}_{\mathbf{u}}+\rho \nabla\left(\mu+V^{\prime}(y)\right)\right) d \mathbf{x} \\
& +\int_{\partial \Omega} J_{b} \cdot \mathcal{B}[\rho] d \sigma+\int_{\partial \Omega} \mathbf{u}_{s} \cdot\left(\tau \mathbf{n}-\mathcal{B}[\rho] \nabla_{s} \rho\right) d \sigma
\end{aligned}
$$

where each term is in the form of a "generalized flux" multiplied by a "generalized force". The first term is the rate of viscous dissipation. The second term is nondissipative (or reversible), from which we obtain

$$
\mathbf{J}_{\mathbf{u}}=-\rho \nabla\left(\mu+V^{\prime}(y)\right) .
$$

For the two boundary terms, under the linear response assumption we get

$$
\begin{aligned}
J_{b} & =-M_{b}(\rho) \mathcal{B}[\rho], \\
-\beta(\rho) \mathbf{u}_{s} & =\tau \mathbf{n}-\mathcal{B}[\rho] \nabla_{s} \rho .
\end{aligned}
$$

To summarize, the diffuse interface model for a single-component fluid consists of the dynamic equations in (3.2), where $\tau$ and $\mathbf{J}_{\mathbf{u}}$ are given in (3.3) and (3.10) respectively; the boundary condition for $\rho$ is given in (3.8) and (3.11a); the boundary conditions for $\mathbf{u}$ consists of (3.11b) and the no-penetration condition $\mathbf{u} \cdot \mathbf{n}=0$.

3.2. Two-component fluids. In the case of two-component fluids, we use the mass fraction of one of the two fluids as the phase variable, and denote it by $\varphi$ $[30,31]$. The total free energy in this case is

$$
\begin{aligned}
E(t)= & \int_{\Omega}\left(\frac{1}{2} \kappa|\nabla \varphi|^{2}+\rho f(\rho, \varphi)+\varphi V^{\prime}(y)\right) d \mathbf{x} \\
& +\int_{\Omega} \frac{1}{2} \rho|\mathbf{u}|^{2} d \mathbf{x}+\int_{\partial \Omega}\left(\gamma_{w f}(\varphi)+\varphi V(0)\right) d \sigma \\
= & E_{p}+E_{k}+E_{s},
\end{aligned}
$$

where the three terms are the potential energy $\left(E_{p}\right)$, the kinetic energy $\left(E_{k}\right)$ and the surface energy $\left(E_{s}\right)$, respectively; $V$ is the surface potential. The equations in the bulk will be written as

$$
\begin{array}{r}
D_{t} \rho=-\rho \nabla \cdot \mathbf{u}, \\
\rho D_{t} \varphi=\nabla \cdot \mathbf{J}_{\varphi}, \\
\rho D_{t} \mathbf{u}=\nabla \cdot \tau+\mathbf{J}_{\mathbf{u}},
\end{array}
$$

where $\tau$ is the viscous stress. As in the one-component case, $\tau$ is assumed to be linear:

$$
\tau=\nu\left(\nabla \mathbf{u}+\nabla \mathbf{u}^{T}\right)+\lambda(\nabla \cdot \mathbf{u}) .
$$


The diffusion current $\mathbf{J}_{\varphi}$, the non-dissipative force from the free energy $\mathbf{J}_{\mathbf{u}}$, and the boundary conditions will be determined below.

Define the chemical potential:

$$
\mu=\partial_{\varphi} f-\frac{\kappa}{\rho} \Delta \varphi
$$

Then using the equations in (3.13), we obtain

$$
\begin{aligned}
\frac{d E_{p}}{d t}= & \int_{\Omega} \rho D_{t}\left(\frac{\kappa}{2 \rho}|\nabla \varphi|^{2}+f(\rho, \varphi)+\frac{\varphi}{\rho} V^{\prime}(y)\right) d \mathbf{x} \\
= & -\int_{\Omega} \mathbf{J}_{\varphi} \cdot \nabla\left(\mu+\frac{1}{\rho} V^{\prime}(y)\right) d \mathbf{x} \\
& +\int_{\Omega} \mathbf{u} \cdot\left(\nabla\left(\rho^{2} f_{\rho}-\frac{\kappa}{2}|\nabla \varphi|^{2}\right)+\nabla \cdot(\kappa \nabla \varphi \otimes \nabla \varphi)-V^{\prime}(y) \nabla \varphi\right) d \mathbf{x}+\int_{\partial \Omega} \kappa \partial_{\mathbf{n}} \varphi \partial_{t} \varphi d \sigma,
\end{aligned}
$$$$
\frac{d E_{k}}{d t}=\int_{\Omega} \rho \mathbf{u} \cdot D_{t} \mathbf{u} d \mathbf{x}=-\int_{\Omega}\left(\nabla \mathbf{u}: \tau-\mathbf{u} \cdot \mathbf{J}_{\mathbf{u}}\right) d \mathbf{x}+\int_{\partial \Omega} \mathbf{u} \cdot \tau \mathbf{n} d \sigma
$$

$\frac{d E_{s}}{d t}=\int_{\partial \Omega}\left(\gamma_{w f}^{\prime}(\varphi)+V(0)\right) \partial_{t} \varphi d \sigma$

On $\partial \Omega, \varphi$ satisfies an equation of the form

$$
\partial_{t} \varphi+\left(\mathbf{u}_{s} \cdot \nabla_{s}\right) \varphi=J_{b}
$$

where $\mathbf{u}_{s}$ is the slip velocity on $\partial \Omega$ and $J_{b}$ is a dissipative contribution yet to be determined. Combining (3.16a), (3.16b), (3.16c) and using (3.17), we obtain

$$
\begin{aligned}
\frac{d E}{d t}= & -\int_{\Omega} \nabla \mathbf{u}: \tau d \mathbf{x}-\int_{\Omega} \mathbf{J}_{\varphi} \cdot \nabla\left(\mu+\frac{1}{\rho} V^{\prime}(y)\right) d \mathbf{x} \\
& +\int_{\Omega} \mathbf{u} \cdot\left(\mathbf{J}_{\mathbf{u}}+\nabla\left(\rho^{2} \partial_{\rho} f-\frac{\kappa}{2}|\nabla \varphi|^{2}\right)+\nabla \cdot(\kappa \nabla \varphi \otimes \nabla \varphi)-V^{\prime}(y) \nabla \varphi\right) d \mathbf{x} \\
& +\int_{\partial \Omega} J_{b} \cdot \mathcal{B}[\varphi] d \sigma+\int_{\partial \Omega} \mathbf{u}_{s} \cdot\left(\tau \mathbf{n}-\mathcal{B}[\varphi] \nabla_{s} \varphi\right) d \sigma
\end{aligned}
$$

where each term is in the form of a "generalized flux" multiplied by a "generalized force". The operator $\mathcal{B}[\varphi]$ in the boundary terms is defined as

$$
\mathcal{B}[\varphi]=\kappa \partial_{\mathbf{n}} \varphi+\gamma_{w f}^{\prime}(\varphi)+V(0) .
$$

The first term in (3.18) is the rate of viscous dissipation. The second term is the rate of energy dissipation due to diffusion. If we assume linear response, then we obtain

$$
\mathbf{J}_{\varphi}=M \nabla\left(\mu+\frac{1}{\rho} V^{\prime}(y)\right) .
$$

The third term is non-dissipative (or reversible), from which we obtain

$$
\mathbf{J}_{\mathbf{u}}=-\nabla\left(p-\frac{\kappa}{2}|\nabla \varphi|^{2}\right)-\nabla \cdot(\kappa \nabla \varphi \otimes \nabla \varphi)+V^{\prime}(y) \nabla \varphi,
$$


where $p=\rho^{2} \partial_{\rho} f$ is the thermodynamic pressure. The boundary conditions for $\varphi$ and $\mathbf{u}_{s}$ are obtained from the last two terms in (3.18):

$$
\begin{aligned}
\partial_{t} \varphi+\left(\mathbf{u}_{s} \cdot \nabla_{s}\right) \varphi & =f_{1}(\mathcal{B}[\varphi]), \\
\tau \mathbf{n}-\mathcal{B}[\varphi] \nabla_{s} \varphi & =f_{2}\left(\mathbf{u}_{s}\right),
\end{aligned}
$$

together with the no-penetration conditions at the wall:

$$
\partial_{\mathbf{n}}\left(\mu+\frac{1}{\rho} V^{\prime}(y)\right)=0, \quad \mathbf{u} \cdot \mathbf{n}=0 .
$$

The functions $f_{1}$ and $f_{2}$ in the above boundary conditions need to be determined by other means, e.g. molecular dynamics simulations. When the two functions are linear and without the surface potential, we recover the generalized Navier boundary condition [14].

To summarize, the diffuse-interface model for two-component fluids consists of the dynamical equations in (3.13), where $\tau, \mathbf{J}_{\varphi}$, and $\mathbf{J}_{\mathbf{u}}$ are given in (3.14), (3.20), and (3.21) respectively, and the boundary conditions in (3.22) and (3.23).

We remark that the philosophy we followed here is different from that of the Onsager's minimum energy dissipation rate principle pursued in [15]. Strictly speaking, Onsager's minimum energy dissipation rate principle should not be considered as a first principle, but rather a mathematical reformulation of the dissipative structure of the model. One has to postulate a specific dissipation function in order to use Onsager's principle. Often this already amounts to assuming the constitutive relation. If the dissipation function is postulated to be quadratic, then one obtains a linear constitutive relation. If the underlying constitutive laws (here the boundary conditions) are smooth, then linear response theory can always be applied in a limited regime as a consequence of Taylor expansion. However, as we have already seen here, the boundary conditions are not necessarily smooth, as in the case of complete wetting. Therefore a more general derivation as we described here is necessary. As we have seen, it is also quite accessible.

Acknowledgement. The work of $\mathrm{E}$ is supported in part by the ONR grant N00014-01-1-0674. The work of Ren is supported in part by the NSF grant DMS0806401 and the Sloan fellowship. We are grateful to an anonymous referee for pointing out an incorrect statement in the draft of the paper.

\section{REFERENCES}

[1] P.G. de Gennes, Wetting: statics and dynamics, Rev. Mod. Phys., 57, 827-863, 1985.

[2] P.G. de Gennes, F. Brochard-Wyart and D. Quere, Capillarity and Wetting Phenomena: Drops, Bubbles, Pearls, Waves, Springer-Verlag, 2003.

[3] E.B. Dussan V, On the spreading of liquids on solid surfaces: static and dynamic contact lines, Ann. Rev. Fluid Mech., 11, 371-400, 1979.

[4] V.M. Starov, M.G. Velarde and C.J. Radke, Wetting and Spreading Dynamics, CRC press, 2007.

[5] Y.D. Shikhmurzaev, Capillary Flows with Forming Interfaces, Chapman \& Hall/CRC, 2008.

[6] D. Bonn, J. Eggers, J. Indekeu, J. Meunier and E. Rolley, Wetting and spreading, Rev. Mod. Phys., 81, 739-805, 2009.

[7] C. Huh and L.E. Scriven, Hydrodynamic model of steady movement of a solid/liquid/fluid contact line, J. Colloid Interface Sci., 35, 85-101, 1971.

[8] E.B. Dussan V. and S.H. Davis, On the motion of a fluid-fluid interface along a solid surface, J. Fluid Mech., 65, 71-95, 1974. 
[9] C. Huh and S.G. Mason, Pressure effects on micellar size, J. Colloid Interface Sci., 81, 396-401, 1977.

[10] L.M. Hocking, A moving fluid interface on a rough surface, J. Fluid Mech., 76, 801-817, 1976.

[11] R.G. Cox, The dynamics of the spreading of liquids on a solid surface. Part 1. Viscous flow, J. Fluid Mech., 168, 169-194, 1986.

[12] P. Seppecher, Moving contact lines in the Cahn-Hilliard theory, Intl J. Engng Sci., 34, 977-992, 1996.

[13] D. Jacqmin, Contact-line dynamics of a diffuse fluid interface, J. Fluid Mech., 402, 57-88, 2000.

[14] T. Qian, X.P. Wang and P. Sheng, Molecular scale contact line hydrodynamics of immiscible flows, Phys. Rev. E, 68, 016306, 2003.

[15] T. Qian, X.P. Wang and P. Sheng, A variational approach to moving contact line hydrodynamics, J. Fluid Mech., 564, 333-360, 2006.

[16] R. Borcia, I.D. Borcia and M. Bestehorn, Static and dynamic contact angles - a phase field modeling, Eur. Phys. J. Special Topics, 166, 127-131, 2009.

[17] P. Yue, C. Zhou and J.J. Feng, Sharp-interface limit of the Cahn-Hilliard model for moving contact lines, J. Fluid Mech., 645, 279-294, 2010.

[18] T.D. Blake and J.M. Haynes, Kinetics of liquid/liquid displacement, J. Colloid Interface Sci., 30, 421-423, 1969.

[19] T.D. Blake, The physics of moving wetting lines, J. Colloid Interface Sci., 299, 1-13, 2006.

[20] J. Koplik, J.R. Banavar and J.F. Willemsen, Molecular dynamics of Poiseuille flow and moving contact lines, Phys. Rev. Lett., 60, 1282-1285, 1988.

[21] J. Koplik, J.R. Banavar and J.F. Willemsen, Molecular dynamics of fluid flow at solid surfaces, Phys. Fluids A, 1, 781-794, 1989.

[22] P.A. Thompson and M.O. Robbins, Simulations of contact-line motion: slip and the dynamic contact angle, Phys. Rev. Lett., 63, 766-769, 1989.

[23] P.A. Thompson, W.B. Brinckerhoff and M.O. Robbins, Microscopic studies of static and dynamics contact angles, J. Adhes. Sci. Technol., 7, 535-554, 1993.

[24] W. Ren and W. E, Boundary conditions for moving contact line problem, Phys. Fluids, 19, 022101, 2007.

[25] S.R. De Groot and P. Mazur, Non-Equilibrium Thermodynamics, Dover Publication; Dover Eds., 1984.

[26] W. Ren, D. Hu and W. E, Continuum models for the contact line problem, Phys. Fluids, Vol 22, 102103, 2010.

[27] J. Israelachvili, Intermolecular and Surface Forces, Academic Press, 1991.

[28] B. Schweizer, A well-posed model for dynamic contact angles, Nonlinear Analysis, 43, 109, 2001.

[29] E.K. Yeh, J. Newman and C.J. Radke, Equilibrium configurations of liquid droplets on solid surfaces under the influence of thin-film forces: Part II. Shape calculations, Colloid Surfaces A, 156, 525-546, 1999.

[30] D.M. Anderson, G.B. McFadden and A.A. Wheeler, Diffuse-interface methods in fluid mechanics, Annu. Rev. Fluid Mech., 30, 139-165, 1998.

[31] J. Lowengrub and L. Truskinovsky, Quasi-incompressible Cahn-Hilliard fluids and topological transitions, Proc. R. Soc. Lond. A, 454, 2617-2654, 1998. 